Voix et Images

voixetimages

\title{
Féminisme et postmodernisme : la Main tranchante du symbole
}

\section{Claudine Potvin}

Volume 17, numéro 1 (49), automne 1991

Louky Bersianik

URI : https://id.erudit.org/iderudit/200943ar

DOI : https://doi.org/10.7202/200943ar

Aller au sommaire du numéro

Éditeur(s)

Université du Québec à Montréal

ISSN

0318-9201 (imprimé)

1705-933X (numérique)

Découvrir la revue

Citer cet article

Potvin, C. (1991). Féminisme et postmodernisme : la Main tranchante du

symbole. Voix et Images, 17(1), 66-74. https://doi.org/10.7202/200943ar d'utilisation que vous pouvez consulter en ligne.

https://apropos.erudit.org/fr/usagers/politique-dutilisation/ 


\title{
Féminisme et postmodernisme: la Main tranchante du symbole
}

\author{
par Claudine Potvin, Université de l'Alberta
}

La Main tranchante du symbole ${ }^{1}$ réunit un ensemble d'essais rédigés entre 1980 et 1990 , à l'exception de quelques extraits de l'Euguélionne (1973) repris pour illustrer une réflexion spécifique sur la langue et de la postface composée en 1978. Ouvrage fragmenté par conséquent, mais dont l'unité réside dans le propos féministe de son auteure: remise en cause du patriarcat à travers une série de commentaires sur la langue, l'écriture, le pouvoir, la tradition religieuse et philosophique, ainsi que l'émergence d'une culture au féminin d'une part; discussion polémique de problèmes concrets et urgents auxquels sont confrontées les femmes d'aujourd'hui de l'autre (la criminalisation de l'avortement, le statut de la maternité face aux nouvelles techniques de reproduction, le sort des mères porteuses, la violence faite aux femmes - viol, abus physique, harcèlement sexuel, discrimination, meurtre -, etc.).

La parution de ce livre en 1990 indique nettement la vigueur de la pensée féministe au Québec au cours de la décennie antérieure en même temps que la résistance qui tend à nier son existence et proclamer sa disparition. En effet, entre l'éclatement des années 1970 et une fin de siècle encore vague, un creux avait semblé s'installer.

Devant le petit écran, remarque Louise Dupré, nous prend la nostalgie des années 1970. L'impression que les femmes de cœur et de tête sont de nouveau mises à l'écart. Où en est le féminisme? Notre grand rêve s'est-il effondré? Sómmes-nous passées au postféminisme, comme nous l'entendons affirmer de plus en plus souvent maintenant? 2

Le problème majeur, ajoute-t-elle, c'est que traditionnellement, la conscience demeure l'apanage de l'homme.

1 Louky Bersianik, la Main tranchante du symbole, Montréal, Les Éditions du Remue-ménage, 1990. Pour toute référence ultérieure à cet ouvrage; j'indiquerai la page entre parenthèses à la suite de la citation.

2 Louise Dupré, "Quatre esquisses pour une morphologie », dans la Théorie, un dimanche, Montréal, les Éditions du Remue-ménage, 1988, p. 122. 
C'est de ce côté que nous assistons à un nouvel effort d'évacuation du féminin, selon Bersianik, à une certaine fatigue * sexuelle * du mâle, québécois ou autre. On voudrait bien ne plus en entendre parler, d'où un certain effet de silence, de voix off, de nouveau dialogue de sourd(e)s. Comme le titre de l'ouvrage l'indique, on s'acharne toujours à trancher dans le vif du sujet/de la sujète. Symbolum, symbole de foi; symbolus, signe de reconnaissance. La main de celui qui officie, celle de Dieu, se reconnaît bien dans le Père et le Fils; le tranchant d'un geste qui écarte, coupe, nie, censure d'un trait la Mère et la Fille. Du côté des femmes donc, conscience de soi court-circuitée, (re)connaissance impossible. Symbolique de la Loi inscrivant le discours du même dans une légalité usurpée, enracinée dans le meurtre de l'autre. De fait, [l'humanité est en deuil de cette amputation symbolique de la moitié de son potentiel (p. 16).

Or, projection d'un fantasme masculin, le postféminisme permettrait sans doute aux hommes de bonne volonté et aux autres de se tenir un discours moins culpabilisant. En ce sens, tour à tour inclus et exclu de la vision et des théories postmodernes, le féminisme s'y encadre d'ailleurs plutôt mal. La brève réflexion qui suit, et qui se situe dans le cadre d'un projet de recherche beaucoup plus vaste sur le féminisme et le postmodernisme en Argentine et au Québec au cours des années 1980 , entend examiner, quoique sommairement, le rapport idéologique entre les deux "mouvements", moments de coexistence, de rencontre ou d'éloignement, à partir des positions critiques de Louky Bersianik.

À ce sujet, l'écrivaine souligne que

modernité et post-modernité - qui concernent bien d'autres domaines que la littérature - sont des catégories définies par des intellectuels mâles, que se sont appropriées les écrivaines et les intellectuelles. Ces notions ont été créées de toutes pièces par les hommes pour leurs propres besoins et là où ils en étaient dans leur histoire littéraire à eux. Je ne suis pas sûre, avoue-t-elle, que les femmes doivent s'y commettre dans les mêmes termes et sur les mêmes terrains, sans risquer de rester dans les marges et d'ajouter à la confusion véhiculée par le masculin, toujours omnipotent dans le cours de la langue et des idées (p. 33).

Cependant, la présence d'une forte voix féministe demeure un des éléments prédominants de la culture postmoderne. Malgré cela, remarque Craig Owens dans son article intitule «The Discourse of Others: Feminists and Postmodernism: ${ }^{3}$, les théoriciens du postmo-

3 Craig Owens, "The Discourse of Others: Feminists and Postmodernism* dans The Anti-Aesthetic. Essays on Postmodern Culture (Ed. et Int. par Hal 
dernisme ont eu tendance à négliger ou réprimer cette voix. L'auteur soutient que, en général, les critiques qui s'intéressent au postmodernisme discutent peu la question de la différence sexuelle et que, par ailleurs, les femmes n'ont guère participé au débat modernisme/ postmodernisme, laissant entendre par là que le postmodernisme serait une autre invention masculine pour exclure les femmes. Andreas Huyssen confirme la position de Owens et réaffirme que la critique féministe s'est tenue éloignée du débat sur le postmodernisme considérant ce dernier sans pertinence pour les préoccupations féministes ${ }^{4}$. Meaghan Morris a montré dans The Pirate's Fiancée. Feminism, Reading, Postmodernism la faiblesse de l'argumentation de Owens ${ }^{5}$. Plus que d'un refus de participer à ce débat, la prétendue absence des femmes témoigne davantage, selon Morris, de leur non-inscription dans l'analyse postmoderne par les critiques euxmêmes (Habermas, Lyotard, Rorty, Jameson, Huyssen, Foster, Owens; etc.), de la lecture indirecte que pratiquent les femmes du phénomène postmoderne et cela à travers la grille féministe (Clément, Cixous, Irigaray, Felman, Gallop, Kofman, Spivak, Jardine, etc.), du dépouillage rapide et limité des textes de femmes par les hommes eux-mêmes, bref de leur oubli pur et simple de nombreuses bibliographies préparées par des critiques masculins.

It would be hard to deny, insiste Morris, that in spite of its heavy (if lightly acknowledged) borrowing from feminist theory, its frequent celebrations of "difference" and "specificity", and its critiques of "Enlightenment" paternalism, postmodernism as a publishing phenomenon has pulled off the peculiar feat of re-constituting an overwhelmingly male pantheon of proper names to function as ritual objects of academic exegesis and commentary. ${ }^{6}$

Représentation d'une pseudo-absence ou acte de reproduction d'un vide, d'une omission volontaire. Maintien du texte et du discours

Foster), Port Townseed, Washington, Bay Press, 1983, p. 57-82. Voir aussi sur le même sujet les deux articles suivants: «Social Criticism without Philosophy: An Encounter between Feminism and Postmodernism * de Nancy Fraser et Linda J. Nicholson et, "Postmodernism and Gender Relations in Feminist Theory * de Jane Flax dans Feminism/Postmodernism (Ed. et Int. par Linda J. Nicholson), New York et London, Routledge, 1990, p. 19-38 et 39-62.

4 Andreas Huyssen, After the Great Divide: Modernism, Mass Culture, Postmodernism, Indiana, 1986, p. 198-199.

5 Meaghan Morris, The Pirate's Fiancée. Feminism, Reading, Postmodernism, London et New York, Verso, 1988.

6. Ibid., p. 12. Traduction: Il serait difficile de nier qu'en dépit de ses emprunts répetés (mais rarement reconnus) aux théories féministes, de ses multiples célébrations de la “différence » et de la « spécificité » et de ses critiques du paternalisme "éclairé ", le postmodernisme, comme phénomène littéraire, a réussi le singulier exploit de reconstituer un imposant panthéon mâle de noms propres qui jouent le rôle d'objets rituels de l'exégèse et du commentaire académiques. 
féminin hors de l'histoire en inscrivant et en écrivant son absence de l'histoire.

Indépendamment de la place qu'on accorde à la culture et à la voix des femmes (critique et fiction), la pensée postmoderne répond certes jusqu'à un certain point à l'esthétique féministe et s'y intègre (danse, peinture, sculpture, architecture, littérature, musique, performances dramatiques, etc.) et il convient peut-être de discuter de la première à partir de la seconde et non le contraire ${ }^{7}$. Dans son introduction à l'essai de Diane Lamoureux sur le féminisme québécois des années 1970, Françoise Collin conclut que celui-ci répond à l'appel postmoderne

quand, court-circuitant la hiérarchie et l'opposition binaire des sexes, il installe partout la différence et l'hétérogénéité, quand il dénonce la confiscation du temps au profit du travail, quand il perçoit le corps comme une surface polymorphe, qui fait peau de partout, quand il définit la lutte des femmes comme un mouvement et non comme une fin totalisante, quand il dénonce l'idée d'une nature féminine (ou masculine) mais aussi bien d'une nature humaine, sans en faire un produit de l'histoire ${ }^{8}$.

L'écriture de Louky Bersianik appartient certes au même souffle. hétérogène: féministe dans son cheminement, postmoderne dans son mode d'opération, en perpétuel mouvement.

De nombreux critiques ont signalé la confusion qui règne dans la définition des termes mêmes. Depuis Lyotard cependant, on s'entend pour confirmer l'importance de sa mise au point dans la Condition postmoderne ${ }^{9}$ et de sa contribution pour la compréhension de la notion même de postmoderne, peut-être plus particulièrement au Québec. Pour ce dernier, la pratique postmoderne se rattache au déclin des métarécits de légitimation; c'est-à-dire à l'incrédulité face à ceux-ci, d'où l'émergence d'un savoir hétérogène qui remet en question les valeurs établies telles que l'humanisme, le progrès, la liberté, la raison, l'autorité, la vérité, etc. Mode de pensée avant tout, le

7 . Il est étonnant que Pierre Milot n'en fasse pratiquement aucune mention dans son livre sur le postmodernisme au Quebec alors qu'il consacre une importante partie de son étude à l'avant-garde des années 1970 (plus particulièrement à la NBJ et aux Herbes rouges) si étroitement liée à l'émergence du mouvement féministe québecois et à des auteures comme Nicole Brossard, France Théret; Louise Dupré, Louky Bersianik, Madeleine Gagnon, etc. Voir la Camera obscura du postmodernisme, Montréal, l'Hexagone, 1988.

8 Françoise Collin, «Le féminisme et la crise du moderne *, introduction à Diane Lamoureux, Fragments et collages. Essai sur le féminisme québécois des années 70, Montréal, les Éditions du Remue-ménage, 1986, p. 10.

9 Jean-François Lyotard, la Condition postmoderne, Paris, Minuit, 1979. Voir aussi du même auteur, le Postmoderne expliqué aux enfants, Paris, Éditions Galilée, 1988. 
postmodernisme appartient également à l'ordre du vécu et représente une attitude, voire une façon d'être, de se concevoir et de créer le monde. Scarpetta reprendra ces notions en termes plus séduisants d'impureté, de corruption, de mixage, de métissage, "réalités" auxquelles la culture des femmes renvoie sans cesse. Il s'agit de sortir de l'ère des ruptures, de remanier le passé et les notions classiques d'histoire, de progrès, de nostalgie. Selon Scarpetta, le refus de l'alternative (entre la régression et le progrès irréversible) et

la volonté d'explorer les zones de coexistence et d'affrontement entre les deux cultures, de les faire s'interpénétrer, réagir, de jouer leur métissage, d'opérer des connexions, des courts-circuits, des recyclages, des détournements, des anachronismes délibérés (autrement dit: de traiter esthétiquement la mutation technique, avec le minimum d'intimidation, le maximum d'aisance et de liberté): c'est la voie postmoderne, celle de l'impureté... Pas de zones préservées, cloisonnées mais des rapts, des transferts, des transpositions, des réappropriations. ${ }^{10}$

Pour les femmes, les écrivaines surtout, ces déplacements s'opèrent dans l'ambiguité, la contradiction. Louky Bersianik considère que la femme est une ambiguïté, science occulte occultée... se découvrant au pluriel (p. 223), déterrée vivante, émergente contre-culturelle. Au niveau textuel, cette ambiguité se manifeste dans la transformation des formes verbales, la réappropriation d'une langue codée d'avance, le vol du signifié par.le jeu sur le signifiant, le déplacement sémantique du Verbe et de la Parole des Pères, de tous les langages étrangers, l'éclatement du symbole entre la dictée de l'inconscient et le tremblement de la conscience (p. 277), l'ouverture et la pluralité des écritures exploréennes, la projection infinie du corps dans la lettre, le mouvement de la main en train de s'écrire, la dissémination du sens.

Dans le contexte québécois, Janet $M$. Paterson a soutenu que le pòstmodernisme se donne précisément dans un texte pluriel, éclaté, parodique, autoréflexif et dans la subversion des codes narratifs (énonciation et énoncé) ${ }^{11}$. Plus que toute autre, l'écriture au féminin, et par extension celle de Louky Bersianik, a cherché à déconstruire les "grands récits " d'origine patriarcale, à * traiter * l'information, la reproduction, l'imitation de l'unique, à transformer le nom/non masculin, à penser à côté pour créer un autre monde (p. 231) d'où émergeraient des avoirs multiples, où circulerait l'être hétérogène, pour que naisse une mémoire du futur et pour [f]aire de l'écriture un rapport amoureux, à ras du corps, un raccord avec le corps du passé et celui du désir d'à présent pour créer une mémoire utilisable (p. 236).

10 Guy Scarpetta, 1Tmpureté, Paris, Grasset, 1985, p. 55 et 59.

11 Voir Janet M. Paterson, Moments postmodernes dans le roman québécois, Ottawa, les Presses de l'Université d'Ottawa, 1990. 
Dans The Postmodern Scene, Kroker et Cook semblent ne retenir qu'une dimension réductrice du féminisme dans sa relation à l'appareil technologique postmoderne. L'industrie des médias, par exemple, aurait tendance à vouer le féminisme à son effritement plus ou moins absolu dans le monde postmoderne en même temps qu'elle considère qu'il représenterait, d'une certaine façon, une possibilité de liberration humaine. What's the relationship of feminist critique to the much-celebrated and perfectly cachet world of postmodernism?, s'interrogent-ils, question à laquelle une des réponses possibles serait que

[t]he fate of feminism in the age of postmodernism is to be a processed feminism: that's the radical danger but also the real promise of feminist critique in technological society. The electronic machine eats up images of women: even (most of all?) emancipation from the patriarchal world of gender ideology is experienced simultaneously as domination and freedom $^{12}$.

La différence sexuelle se trouve ainsi parallèlement médiatisée dans le rejet et la consommation (nausée et cannibalisme), là coupure schizophrénique et la violence, l'extase et la cruauté, le sado-masochisme, le malaise et la corruption, entre les restes d'un musée en ruines et les signes flottants de la séduction et de la jouissance, entre la pourriture du/des corps et la mort du sexe. Toutefois, la libération ne peut se vivre dans lillusion maintenue par le dominant. La violence a un sexe, la violence a un système bien établi qui est le patriarcat et [q]uand la domination est menacée, la violence reprend ses droits (p. 164).

Bien plus, il serait commode, ajoute Louky Bersianik,

de considérer la post-modernité comme un film projeté à l'envers, où les failles volontaires de la modernité se colmateraient à ses occlusions, où la femme naguère fragmentée et in-signifiante se lirait toute entière non plus entre les lignes, mais outrageusement visible dans son intégralité et son intégrité (p. 32).

12 Arthur Kroker et David Cook, The Postmodern Scene. Excremental Culture and Hyper-Aesthetics, Montrél, New World Perspectives, 1986, p. 21-22. Traduction de la question: Quelle est la relation de la critique féministe avec l'univers très acclamé et très particulier du postmodernisme? Traduction de la reponse: Le destin du féminisme d̀ l'áge du posmodernisme est d'étre un féminismé remâché: c'est le danger le plus grave qu'il court, mais c'est en même temps le seúl espoir de la critique féministe dans une société technologique. La machine électronique gobe l'image des femmes: même (surtout?) l'émancipation de l'«ideology of gender" par rapport au monde patriarcal est vécue d la fois comme domination et comme liberte. 
Mais comme l'auteure le dit encore, nous n'en sommes pas au postpatriarcat et une véritable culture au féminin doit, pour permettre une autonomie totale, " remâcher* des images de femmes transmises par un système aliénant comme on mastique ses mots avant de les régurgiter afin de * trancher * à son tour dans le symbolique et.le langage masculin.

Si la femme n'a pas de nom et ne peut occuper l'espace de la signature dans le texte institutionnel, si le masculin l'emporte toujours sur le féminin et que les académiciens continuent de l'ignorer, si la langue ment et renvoie encore à un discours premier, unique, autoritaire, original qui n'autorise qu'une mauvaise photo(copie), une simple reproduction, un calque ou pis encore, une piètre imitation, la femme qui écrit ne peut que menacer et « subversionner» le monde comme le suggère Louky, en faire le procès dans un long processus qui va de la non-existence à l'existence multipliée, multiple, immense, douée d'une puissance qui ne s'était jamais vue (p. 279).

Toute la démarche de Louky Bersianik, résumée dans les textes de la Main tranchante du symbole échelonnés sur une période de dix ans comme je l'ai indiqué auparavant, signale cette coupure épistémologique opérée par les femmes et l'émergence d'un autre discours, différent de celui qui est resassé depuis Platon. Ce nouveau discours se veut construit à partir d'une mémoire du futur, palimpseste de son propre imaginaire (celui de la femme) tenu dans l'oubli, toujours voué au déni, toujours au mépris (p. 189) et suit de près le décloisonnement postmoderne ou le contient en germes. Dans son recueil, Bersianik remet en question l'esthétique et la philosophie occidentales qui ont systématiquement éliminé la femme de leur champ de vision et qui continuent de se montrer foncièrement intolérantes envers la pensée féministe. Elle s'y livre à une pratique de déconstruction au sens derridien et au sens où Caroline Bayard la définit: Deconstruction, écrit cette dernière, implies not only a breaking down of textual constituents, an explosion, a dispersion over the page, but also a dissemination of meanings which then turn into undeterminable, unstable, shifting quantities. ${ }^{13}$

Dans ses essais, l'auteure met également en place une sémiotique du sujet femme qui tend à renverser le sens patriarcal et à changer le sens "apparent *. Car il fut une époque où les pères n'existaient pas (p. 184), y lit-on, et les femmes sont des émergentes contre-culturelles (p. 223), impures, obscènes, parce que nées de femmes, d'étrangères

13 Caroline Bayard, The New Poetics in Canada and Quebec. From Concretism to Post-Modernism, Toronto, University of Toronto Press, 1989, p. 182. Traduction: La déconstruction implique non seulement un effondrement des constituants textuels, une explosion, une dispersion hors de la page, mais aussi une dissémination des significations qui deviennent alors des quantités indéterminables, instables, changeantes. 
(p. 128), récalcitrantes et rebelles devant la table d'écriture chargée de faire trembler la terre pour que s'y installe le MOI de nos mémoires sur le TOIt de l'histoire pour le faire s'effondrer. My mory instead of his story (p. 251). Selon Caroline Bayard, l'histoire comme reproduction; remise en acte, réémergence, plus que comme pur reflet des événements passés, contient les moments postmodernes des cultures francophone et anglophone (québécoise et canadienne), et c'est ce qui distinguerait la modernité du postmodernisme.

History is on our threshold, suggère-t-elle, and it is made of many stories, hers and yours. Its threads will be woven together, by many hands, in a multi-layered process. No unitary voice speaks from its components, but the many voices and the many whispers which have long been silenced and now emerge to question the very possibility of totality and finality. Theirs is an open, in-process history: scribes at work, scribes who listen and share their voices. 14

Louky Bersianik a fait sienne cette conception de l'histoire en continuant d'aménager dans ses fictions des fenêtres qui assurent une meilleure circulation de l'air, cherchant à faire entendre des voix différentes, décentrées, des harmonies et des accords variés, à multiplier, déplacer et recréer le sens ailleurs que dans l'absolu, à se donner des sexualités ouvertes, plurielles, hétérogènes, à démembrer la symbolique figée et stérile du patriarcat. Écriture vécue comme une dynamique, une gestuelle qui permet de concevoir le féminisme comme un espace de discussions, de remises en questions, de changements, [...] un territoire mouvant, ouvert, multiforme et en mouvement ${ }^{15}$, bref un lieu postmoderne.

Mouvement de l'œuvre d'art éternellement transitoire qui ne "peut» durer, qui ne vaut et ne se veut que pour un coup d'œil de la spectatrice ou de la consommatrice dans la vitrine. Ainsi cette " robe de chair" de Jana Sterback (Vanitas: Flesh Dress for an Albino Anorexic) exposée à la Galerie Nationale du Canada au printemps dernier, œuvre sans cesse à recréer. Constamment à redire, le texte refuse de signifier une fois pour toutes. Faite de viande crue et pendue à un cintre, en perpétuelle décomposition (le corps; la peau: femme-mannequin, femme-robot accrochée à l'armature de l'autre), toujours renouvelée (les tranches de bœuf frais sont épisodiquement

14 Caroline Bayard, op. cit., p. 195. Traduction: L'histoire est à notre porte et elle est constituée d'existences multiples, les leurs comme les vôtres. Ses fils seront tissés par plusieurs mains, dans une démarche en plusieurs étapes. Il n'y a pas de voix unique qui monte de ses composantes, mais les voix et les chuchotements multiples qui sont longtemps restées silencieux et se font maintenant entendre pour interroger la possibilité rélle de totalité et de finalite. C'est l'histoire en marche: ecrivaines au travail, vous qui entendez et partagez leurs voix.

15 Louise Dupre, op.cit., p.133. 
remplacées), cette robe-femme à *croquer * renvoie entre autres au paradigme de la nourriture dans la société contemporaine qui "dévore * les femmes obsédées par le poids (* deux poids deux mesures "), la ligne (la ligne du *bon * sens à ne pas dépasser), la diète (la privation, l'ordre d'un * régime * à suivre, la conduite acceptable). Entre la boulimie et l'anorexie, les femmes se vêtent et se dévêtent depuis des millénaires de la peau des autres comme elles se par(l)ent dans/de la langue du Père. Marqué de la griffe du grand couturier, de l'artiste, le vêtement (la création) ne s'en désintègre pas moins sous le regard des passant(e)s. Le sens se déshabille, circule d'un buste à l'autre, se recouvre d'une nudité qui laisse deviner un autre corps que l'artiste même refuse de s'approprier.

Alors la modernité? Pourrions-nous conclure en reprenant l'interrogation de Louky Bersianik et en y ajoutant celle du postmodernisme, OUI si procès de la signifiance, si questionnement des codes, si refus de la symbolique patriarcale, si biographie du cerveau (Barthes), si pratique furieuse d'intelligence, si conscience des fausses rationalités, si exploration de l'irréel, du langage, si intention profonde et passion durable (p. 24). 